\title{
Homeopathy and cancer: a literature review
}

\author{
Marina das Neves Gomes, Carla Holandino \\ Federal University of Rio de Janeiro. UFRJ, Brazil
}

\begin{abstract}
The World Health Organization states that deaths from cancer are increasing reaching an estimated 12 million deaths by 2030 [1]. In Brazil will be about 480,000 new cases by the end of 2011 [2]. For the treatment of cancer conventional therapies are used, such as chemotherapy[3] and radiotherapy [4] which can cause many adverse reactions. Complementary therapies such as homeopathy can be combined to traditional cancer treatment with the aim of minimizing these adverse reactions, relieving the symptoms of the disease itself and its treatment. Other complementary therapies which can aid in cancer treatment are electrotherapy, acupuncture and electroacupuncture, nutritional supplements, probiotics, phytotherapy, among others [5]. The literature shows the homeopathic medicines can be prescribed using the patient's physical constitution as one strategy to help cancer patients [6-11]. This work aims to compile the literature review done with in vitro and in vivo models describing the mechanisms of action of homeopathic medicines used to treat different kinds of cancer. The research findings showed that homeopathy can help the patient to come to terms with the disease, after cancer diagnosis which often brings with itself denial, fear and a host of psychological disorders that cause an unbalance in the body. In addition, some works done with in vitro and in vivo models have shown that homeopathic medicines can modulate the immune system, activating macrophages and inducing the release of cytokines. These and other effects may help the body to overcome the cancer. The use of homeopathic therapy as complementary to conventional cancer treatment has promising results and should be further investigated.
\end{abstract}

Keywords: Homeopathy; Cancer; Complementary Therapies; Homeopathic Medicines.

[1] World Health Organization - WHO. Cancer [online]. c2011. Available from: http://www.who.int/mediacentre/factsheets/fs297/en/

[2] Instituto Nacional do Câncer - INCA, Câncer [online]. Brasil: Ministério da Saúde. c2010 Available from: http://www.inca.gov.br/regpop/2003/index.asp?link=conteudo_view.asp\&ID=10

[3] Feng S, Chien S. Chemical Engineering Science. 2003; 58 (18): 4087-4114.

[4] National Institutes of Health [online]. USA: National Cancer Institute. c2010. Available from: http://www.cancer.gov/cancertopics/what-is-cancer

[5] National Center for Complementary and Alternative Medicine - NCCAM [online]. USA: Health Info. Available from: http://nccam.nih.gov/health/cancer/camcancer.htm

[6] Vozianov AF, Simeonova NK. British Homoeopathic Journal. 1990; 79: 148-151.

[7] Thompson EA. Complementary therapies in nursing \& midwifery. 1999; 5: 37-41.

[8] Varricchio MCBN, Pinto LF, Andrade EM, Pellagio SS. Revista Homeopatia Brasileira. 2000; 6 (1): 64-67. 
[9] Schlappack O. Homeopathy. 2004; 93: 210-215.

[10] Thompson EA, Reilly D. A prospective observational study. Homeopathy. 2003; 92: 131-134.

[11] Milazzo S, Russell N, Ernst E. European Journal of Cancer. 2006; 42: 282-289.

\section{Homeopatia e câncer: uma revisão da literatura RESUMO}

A Organização Mundial de Saúde (OMS) adverte que as mortes por câncer estão aumentando a cada ano. Até 2030 a OMS estima que o número de mortes por câncer deva chegar a 12 milhões [1]. No Brasil serão cerca de 480.000 novos casos até o final de 2011[2]. Para o tratamento do câncer são usadas terapias convencionais, tais como a quimioterapia[3] e a radioterapia[4] que podem causar muitas reações adversas. Terapias complementares, como a homeopatia, podem ser combinadas ao tratamento tradicional do câncer, com o objetivo de minimizar estas reações adversas, aliviando os sintomas da própria doença e do tratamento. Outras terapias complementares que podem auxiliar no tratamento do câncer são a eletroterapia, a acupuntura e a eletroacupuntura, os suplementos nutricionais, os probióticos, os fitoterápicos, dentre outras[5]. A literatura mostra que os medicamentos homeopáticos podem ser prescritos usando a constituição física do paciente como uma estratégia para ajudar pacientes com câncer[6-11]. O presente trabalho tem como objetivo compilar os principais artigos publicados na literatura realizados com modelos in vitro e in vivo, que descrevem os mecanismos de ação dos medicamentos homeopáticos utilizados para o tratamento de diferentes tipos de câncer. Os resultados da pesquisa mostraram que a homeopatia pode ajudar o paciente na aceitação da doença, após o diagnóstico de câncer. Este indivíduo muitas vezes traz consigo o medo, a negação e uma série de distúrbios psicológicos que se desenvolvem após a descoberta da doença, os quais podem causar um desequilíbrio no organismo. Além disso, estes trabalhos vêm mostrando que os medicamentos homeopáticos podem modular o sistema imunológico, ativando macrófagos e induzindo a liberação de citocinas. Estes e outros efeitos podem ajudar o organismo a superar o câncer. Desta forma, o uso da homeopatia como tratamento complementar ao tratamento convencional do câncer vem apresentando resultados promissores que merecem ser investigados.

Palavras-chave: Homeopatia; Câncer; Terapia Complementar; Medicamentos Homeopáticos.

\section{(c)) BY-NC-ND Licensed to GIRI}

Support: authors declare that this study received no funding

Conflict of interest: authors declare there is no conflict of interest

Correspondence author: Marina das Neves Gomes, marina.neves.gomes@gmail.com

How to cite this article: Gomes MN, Holandino C. Homeopathy and cancer: a literature review. Int J High Dilution Res [online]. 2011 [cited YYYY Month dd]; 10(36): 145-146. Proceedings of the XXV GIRI Symposium and VIII CBFH; 2011 Sep 04-07; Foz do Iguaçu (Brazil). GIRI and ABFH; 2011; Available from: http://www.feg.unesp.br/ ojs/index.php/ijhdr/article/view/483/496 